\title{
Exported oscillator competition: A concept to analyze complex rhythms
}

\author{
A. M. Lacasta, ${ }^{1}$ J. M. Sancho, ${ }^{2}$ and F. Sagués ${ }^{3}$ \\ ${ }^{1}$ Departament de Física Aplicada, Universitat Politècnica de Catalunya, Avinguda Gregorio Marañon 44, E-08028 Barcelona, Spain \\ ${ }^{2}$ Departament d'Estructura i Constituents de la Matèria, Universitat de Barcelona, Diagonal 647, E-08028 Barcelona, Spain \\ ${ }^{3}$ Departament de Química Física, Universitat de Barcelona, and Diagonal 647, E-08028 Barcelona, Spain
}

(Received 23 May 2005; published 10 January 2006)

\begin{abstract}
We study the interaction between two independent nonlinear oscillators competing through a neutral excitable element. The first oscillator, completely deterministic, acts as a normal pacemaker sending pulses to the neutral element which fires when it is excited by these pulses. The second oscillator, endowed with some randomness, though unable to make the excitable element to beat, leads to the occasional suppression of its firing. The missing beats or errors are registered and their statistics analyzed in terms of the noise intensity and the periods of both oscillators. This study is inspired in some complex rhythms such as a particular class of heart arrhythmia.
\end{abstract}

DOI: 10.1103/PhysRevE.73.016206

PACS number(s): 05.45.Xt, 05.40.-a, 89.75.Kd

Synchronization of nonlinear oscillators, either mutual or entrained to an input signal, has been and continues being a very active topic of research [1]. However, intrinsically different, at least from a formal point of view, is the issue of an "external" or "exported" competition between a pair of oscillators with distinct and arbitrary frequencies. By exported, we mean that the competition is assumed to take place and is being registered in a neutral third excitable element. Although this scenario has been much less investigated than standard synchronization, recently published related approaches are those of Balenzuela and Garcia-Ojalvo [2], when analyzing in a neuronal context binaural pitch perception, and of Soen et al. [3], dealing with spontaneous rhythm disorders in self-assembled networks of heart cells. Notice also from the very definition of the problem that we will refer here to purely dynamical units without any consideration of spatial degrees of freedom. In this respect, we completely leave apart competition of oscillatory pacemakers in extended systems as they are common in optical or chemical self-organizing systems.

Our model is organized hierarchically, i.e., one of the oscillators is dominant, making the neutral excitable element to fire whenever possible, whereas the second one just perturbs it, leading to its anticipated activation and/or refractoriness. The outcome is thus a signal from the excitable element with the underlined periodicity of the dominant pacemaker, but is sporadically punctuated with missing pulses. To our understanding, this description is fundamentally equivalent, but simpler to analyze than the apparently more natural starting assumption based on two nonhierarchical oscillators, i.e., each of them equally able to ignite the excitable element. On the other hand, the dynamical scheme constructed in this way is rendered a little bit more versatile and realistic by considering a certain degree of stochasticity in its operational rules. In this respect, we introduce a noise term in the equations of the second oscillator preventing its perfect periodic perturbation of the slaved excitable element.

Although our main interest is to formulate a simple and generic model for a somewhat new concept, that of the exported competition of two hierarchical oscillators as defined above, our study is somewhat inspired by the particular class of cardiac arrhythmia called pure ventricular parasystole [4]. Essentially, it amounts to a cardiac disordered rhythm caused by the interaction of two competing and largely independent oscillators [5]. One is the normal pacemaker, referred to as sinus or sinoatrial node. The second one is an ectopic focus located in the ventricles, firing with a larger and more irregular period than the sinoatrial node. A third neutral excitable element could mimic the whole ventricular tissue whose signals are monitored. Systematic statistical studies of such records have appeared recently in the physics literature [6,7].

Our approach is based on models presenting saddle-node bifurcation phenomenology. The generic activator-inhibitor equations read,

$$
\epsilon \frac{d u}{d t}=u-\frac{u^{3}}{3}-v+I, \quad \frac{d v}{d t}=g(u)-v+\xi(t),
$$

where $u$ and $v$ denote the activator and inhibitor adimensional variables, and the function $g(u)$ has the form,

$$
g(u)=A e^{B u},
$$

with $A=0.2$ and $B=3.0$. This choice reproduces the nullclines of the Morris-Lecar type I model [8-10]. $\xi(t)$ would represent a Gaussian white noise of intensity $D$, with zero mean and correlation,

$$
\left\langle\xi(t) \xi\left(t^{\prime}\right)\right\rangle=2 D \delta\left(t-t^{\prime}\right) .
$$

The parameter $I$ represents the external input and it controls the regime, oscillatory or excitable, of this model (oscillatory for $I>2 / 3$ and excitable otherwise). The function $g(u)$ [Eq. (2)] has been chosen to have nullclines that intersect each other as shown in Fig. 1.

In the oscillatory regime, the model allows us, by changing $I$, to control the period of firing in a quite wide range. In Fig. 2, we represent the period, as a function of $I$, for the deterministic case $(D=0)$. It is remarkable that, in spite of the important differences obtained in the period, the duration and shape of the pulses remain almost unchanged, as the inset of Fig. 2 shows for two very distinct periods.

The addition of noise, apart from introducing a certain 

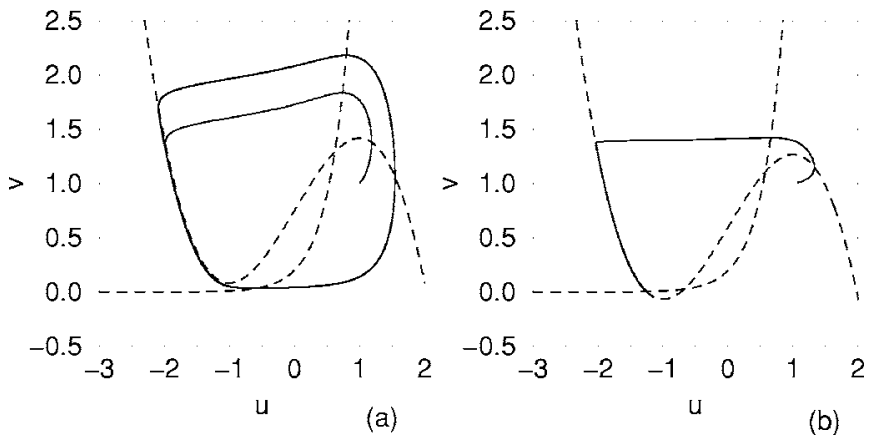

FIG. 1. Nullclines (dashed lines) and $v-u$ trajectories (solid lines) of oscillatory $I=0.68$ (a) and excitable $I=0.6$ (b) element.

degree of irregularity in the time between pulses, leads to a decrease in the averaged firing period. The induced irregularity can be measured by the normalized variance, $R$ $=\sqrt{\left\langle\left(\Delta t_{p}\right)^{2}\right\rangle} /\left\langle t_{p}\right\rangle$, where $t_{p}$ are the interpulse intervals and $\left\langle\left(\Delta t_{p}\right)^{2}\right\rangle$ its variance. In Fig. 3, we represent $R$ and the period $T=\left\langle t_{p}\right\rangle$ as a function of the noise intensity. The monotonous increase of $R$ with respect $D$ is a signature of the oscillatory regime under noise.

Figure 3 can allow us to choose the adequate range of noise values in our study. We will concentrate on a region, from $D=10^{-6}$ to $D=10^{-5}$ characterized by very small differences between periods $T$, but a quite important variability in $R$. This allows us to focus on the effects of the randomness of the perturbing oscillators but with a very good control of its mean period.

The explicit model consists of two nonlinear oscillators whose signals are exported to a neutral excitable element where they compete. Each of these three elements are essentially modeled by Eqs. (1)-(3), but with appropriate parameters for each one.

The normal pacemaker $S$ produces the standard periodic pulses and follows the equations:

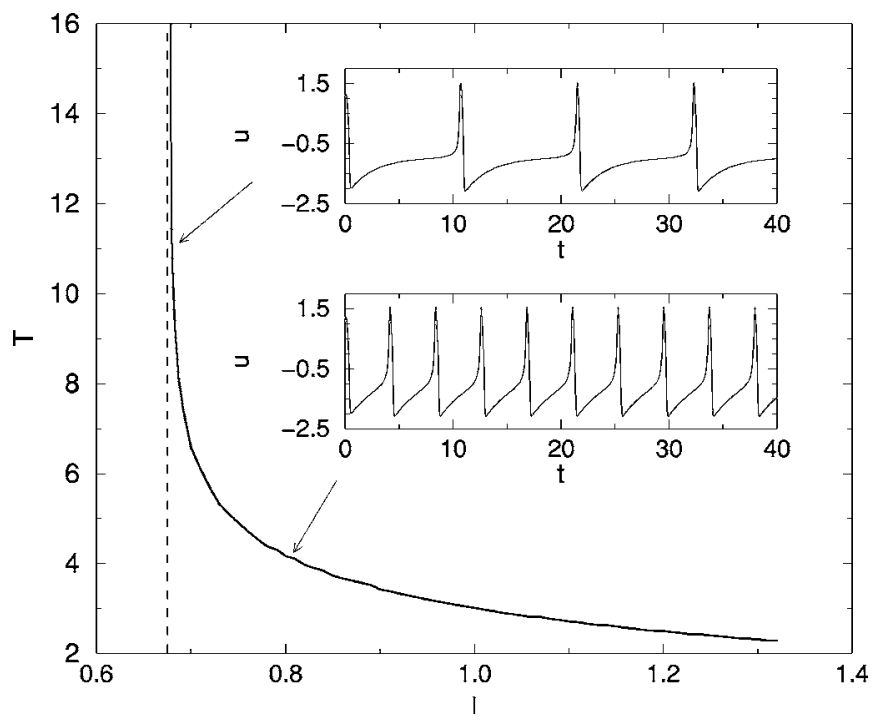

FIG. 2. Period of firing for an oscillatory element as a function of the parameter $I$, corresponding to the deterministic case $(D=0)$ and parameter $\epsilon=0.1$. Insets: Dynamical evolution for $I=0.68$ (top) and 0.8 (bottom).

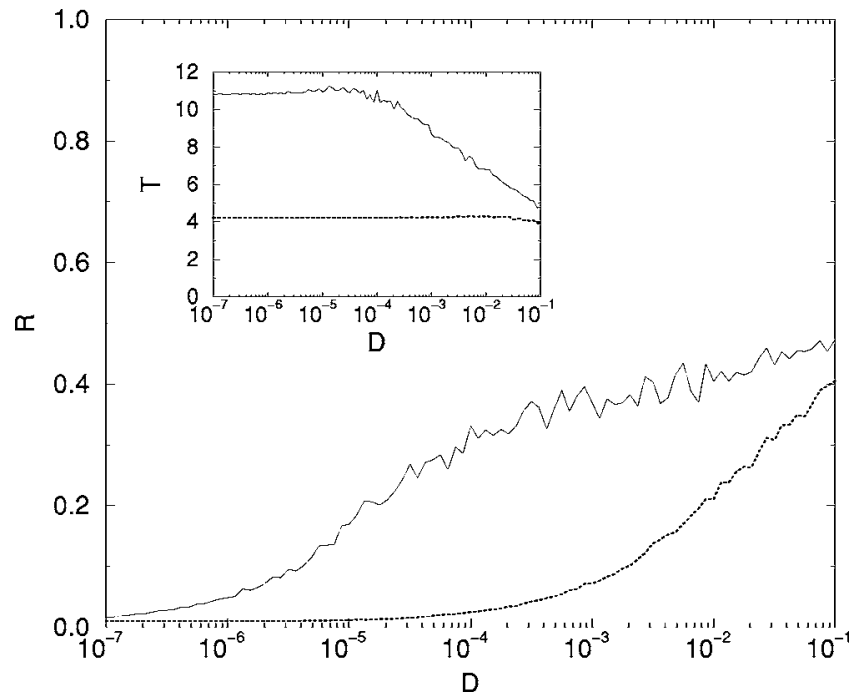

FIG. 3. Normalized variance $R$ and averaged period $T$ (inset) as a function of the noise intensity. They correspond to parameters $I=0.68$ (solid lines) and 0.8 (dotted lines).

$$
\begin{gathered}
\epsilon_{S} \frac{d u_{S}}{d t}=u_{S}-\frac{u_{S}^{3}}{3}-v_{S}+I_{S}, \\
\frac{d v_{S}}{d t}=g\left(u_{S}\right)-v_{S},
\end{gathered}
$$

with $I_{S}>2 / 3$ (oscillatory with period $T_{S}$ ).

The ectopic focus $E$ is characterized by a longer and more irregular period thus incorporating the noise,

$$
\begin{gathered}
\epsilon_{E} \frac{d u_{E}}{d t}=u_{E}-\frac{u_{E}^{3}}{3}-v_{E}+I_{E}, \\
\frac{d v_{E}}{d t}=g\left(u_{E}\right)-v_{E}+\xi(t),
\end{gathered}
$$

with $I_{S}>I_{E}>2 / 3$ (oscillatory with period $T_{E}>T_{S}$ ).

The excitable element is simultaneously excited by the normal pacemaker and perturbed by the ectopic pulses. Its dynamics follows:

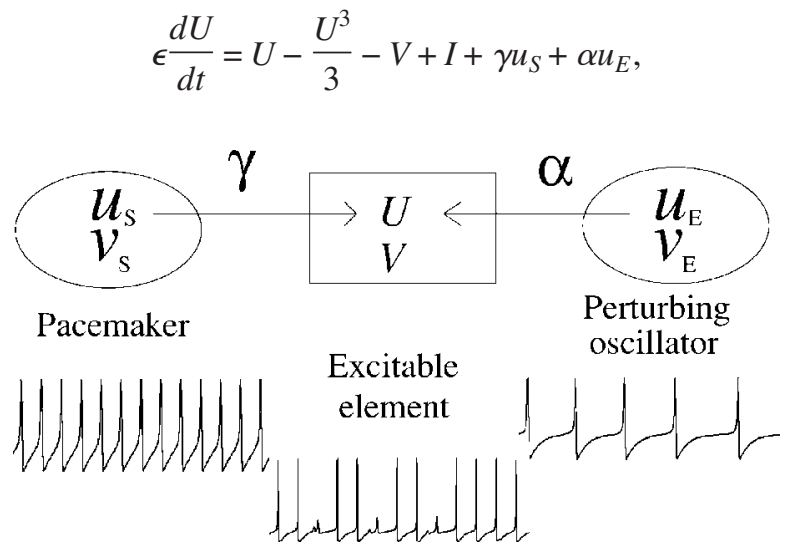

FIG. 4. Schematic diagram of the proposed coupling to simulate the considered oscillator competition. 

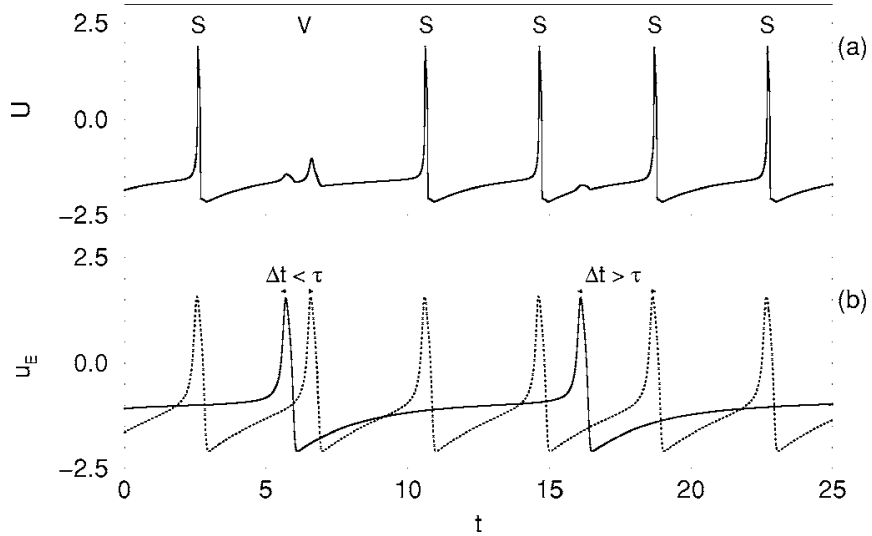

FIG. 5. (a) Train of pulses $U$ for parameters: $\gamma=0.2, \alpha=0.1$, $I_{S}=0.82\left(T_{S} \approx 4\right), I=0.6$ (excitable), $I_{E}=0.68\left(T_{E} \approx 11\right), \epsilon_{S}=\epsilon_{E}=0.1$, $\epsilon=0.01$, and $D=10^{-6}$. (b) Ectopic input $\left(u_{E}\right)$ for the record in (a). Normal input $\left(u_{S}\right)$ is also shown (dotted line).

$$
\frac{d V}{d t}=g(U)-V
$$

with $I<2 / 3$ (excitable). Both normal and ectopic inputs are introduced in the activator equation through the coupling terms, $\gamma u_{S}$ and $\alpha u_{E}$, respectively. In Fig. 4, a diagram of the different elements and an example of their signals are presented.

We have numerically integrated the set of Eqs. (4)-(6), with the function $g\left(u_{i}\right)$ chosen according to Eq. (2). In general, the combined action of the two oscillators on the excitable element produces a train of pulses (beats) with period $T_{S}$, but with some missing beats (arrhythmia). An example is shown in Fig. 5. Defining $\Delta t$ as the time interval between an ectopic beat and the next normal beat, we observe, in the
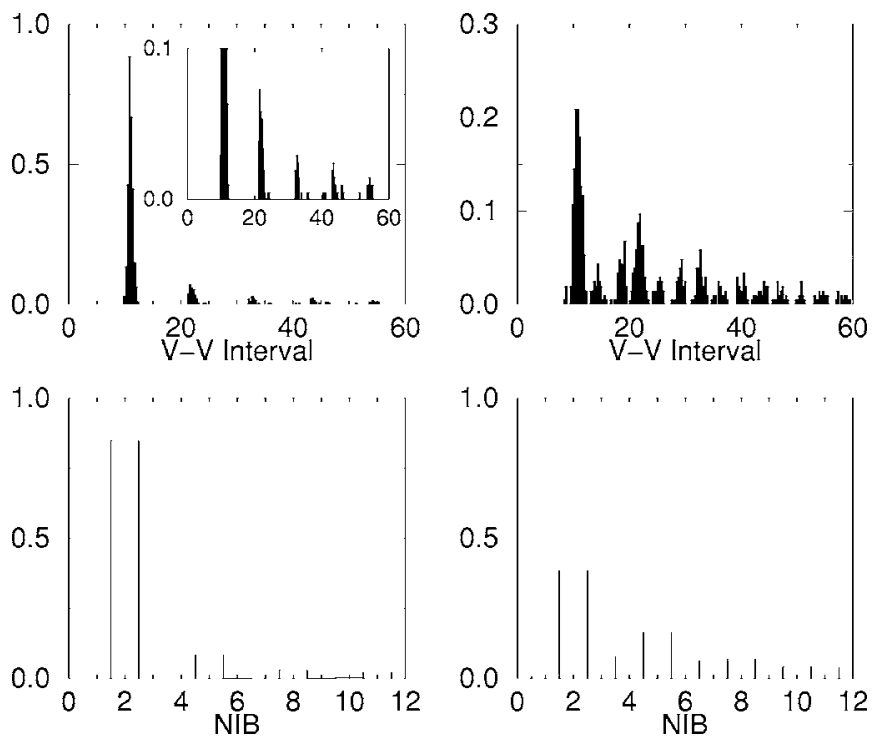

FIG. 6. Normalized $V-V$ interval histogram and NIB histogram for noise intensities: $D=10^{-6}$ (left column) and $D=10^{-5}$ (right column). Parameters $I_{S}$ and $I_{E}$ have been chosen to have $T_{S}=3.6$ and $T_{E}=10.8$. Other parameters are those of Fig. 5 .
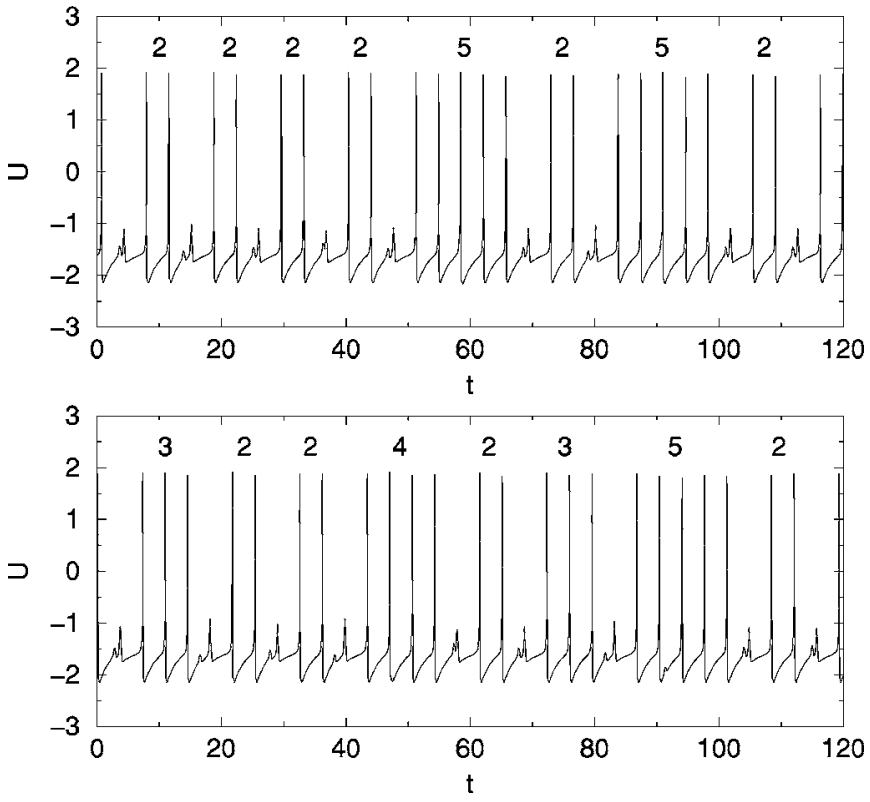

FIG. 7. Sequence of pulses corresponding to the cases of Fig. 6. $D=10^{-6}$ (upper panel) and $D=10^{-5}$ (lower panel). NIB sequences are pointed out.

panel (b) of this figure, how an ectopic pulse produces the inhibition of a normal one if $\Delta t$ is smaller than some characteristic time $\tau$. This time $\tau$ does depend on the characteristics of the excitable element and the coupling parameters $\alpha$ and $\beta$. One expects that, if the pacemaker period $T_{S}$ is equal or smaller than $\tau$, all ectopic pulses would be effective. This point will be confirmed later on.

The following definitions are worth introducing. A $S$ beat will denote an expressed pulse coming from the excitable element due to the firing of the $S$ oscillator. A $V$ beat, on the other hand, will correspond to the trace of a missing or unexpressed $S$ beat in the output signal of the excitable element (see Fig. 5). In order to analyze the sequence of pulses, we evaluate two important characteristics: the $V$ - $V$ interval, which is the time between two $V$ beats, and the NIB, which is the number of $S$ beats between two consecutive $V$ beats.

These two characteristics have been analyzed from long sequences of $N=20000$ pulses for several period ratios and noise intensities. For the determistic situation $(D=0)$, we observe, in general, the presence of only three values of NIB. For example, for $T_{E} / T_{S}=3.2, \mathrm{NIB}=2,9,12$. These NIB triplets depend on the period ratio, but always agree with the

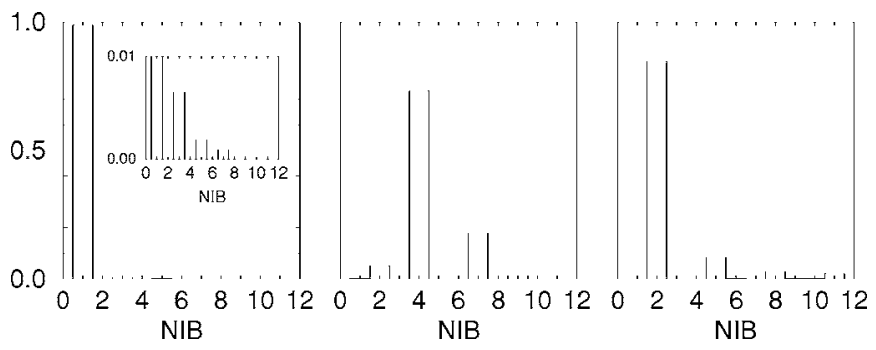

FIG. 8. Normalized NIB histograms for noise intensity $D=10^{-6}$ and ratio $T_{E} / T_{S}=2,2.5$ and 3 , from left to right. 


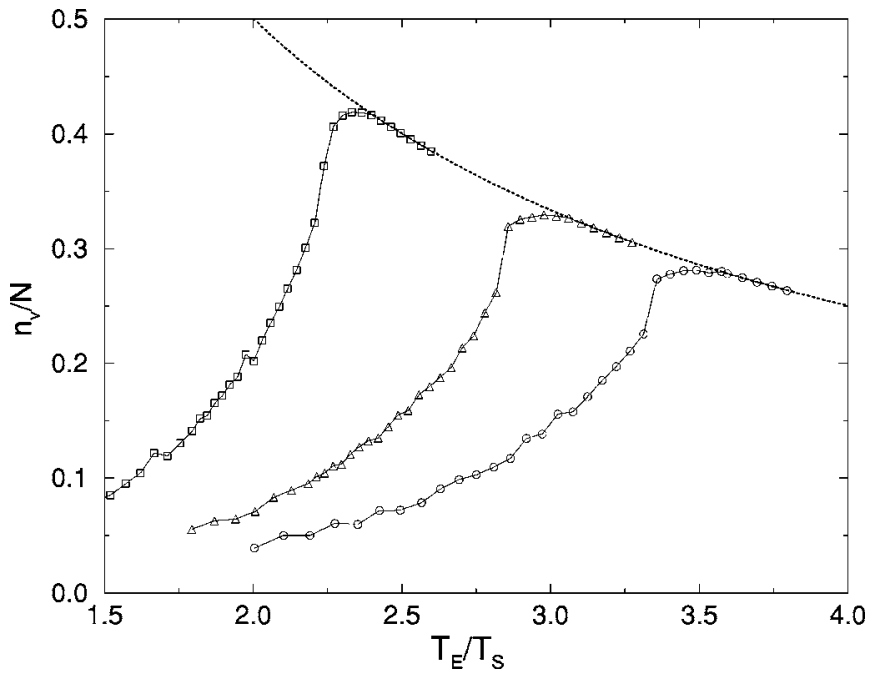

FIG. 9. Relative error versus period ratio $T_{E} / T_{S}$ for $T_{E}=10.8$ (circles), 9.2 (triangles), and 7.2 (squares). $T_{S}$ is changing from 2.8 to 5 , approximately. $D=10^{-6}$ and other parameters are those of previous figures. Dotted line represents the limit condition $n_{V} / N=T_{S} / T_{E}$.

rules obtained from circle map models [4]. For $T_{E} / T_{S} \approx 3$, only the value $\mathrm{NIB}=2$ is present. This situation changes when some noise is included. In Fig. 6, we present the normalized histograms of the $V-V$ interval and the NIB, for $T_{E} / T_{S} \approx 3$ and two different values of the noise intensity. For the lower value of noise, the histogram of the $V-V$ intervals shows a set of equidistant peaks reproducing multiples of $T_{E}$. We also observe that only some NIB values are present: 2,5 , $8,11, \ldots$. For a higher value of noise, the $V-V$ intervals histogram presents a quite different and less defined structure. Correspondingly, many other values of NIB appear in the record but the particular indices 2, 5, $8 \ldots$ still present relative maxima. It is thus clear that the role of the noise is to broaden the NIB histogram. This important difference for both noise intensities is also apparent in the sequences of pulses shown in Fig. 7. On the other hand, for a fixed value of noise intensity, the NIB histogram does depend on the period ratio $T_{E} / T_{S}$, as can be seen in Fig. 8, where some different number sequences are observed.

The effectiveness of ectopic beats can be measured by the relative error, evaluated as the missing beats over total ones (either expressed or unexpressed). In spite of the different rhythms shown in Fig. 7, one can check by simple pulse counting that the amount of errors for both $D$ values is essentially the same. This fact has been confirmed by analyzing longer simulations. Thus the error rate does not depend very much on the noise intensity, but indeed on the relative period of normal and ectopic beats. In order to study further this behavior, we have fixed the period $T_{E}$ and have varied $T_{S}$. Results are shown in Fig. 9, for three different values of $T_{E}$. It is easy to see that, when $T_{S} \leqslant \tau$, all ectopic inputs are effective, and then the relative error has to be $n_{V} / N=T_{S} / T_{E}$. Figure 9 shows that this situation is reached, as anticipated, for low enough $T_{S}$. From this picture, we can estimate the time $\tau$ using any of the three cases, and a value of $\tau \approx 3$ is obtained for this set of parameters. In order to analyze the

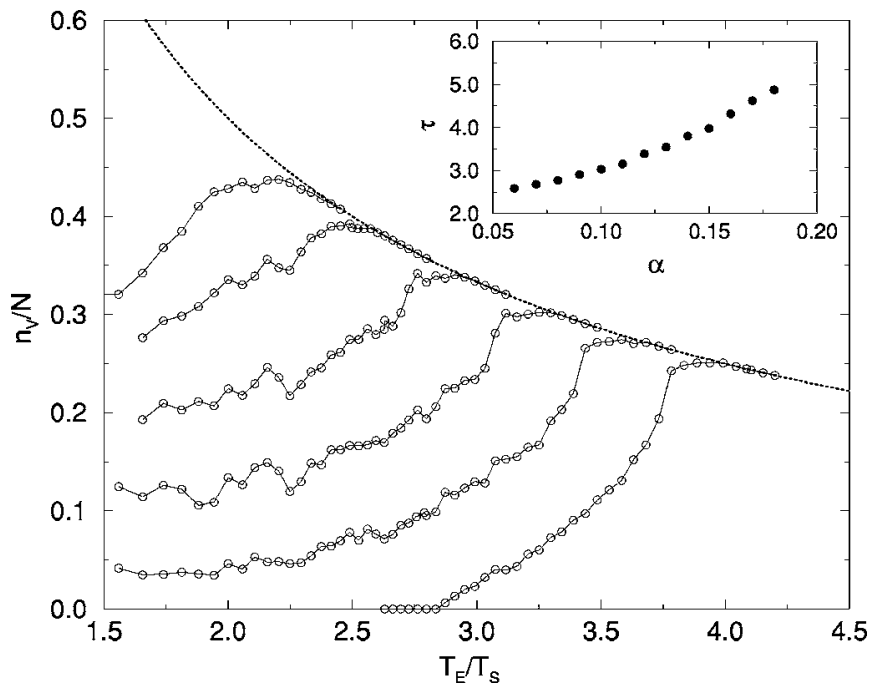

FIG. 10. Relative error versus period ratio $T_{E} / T_{S}$ for $T_{E}=11$, $D=10^{-5}, \gamma=0.2$, and the coupling parameter $\alpha$ going from 0.08 (bottom curve) to 1.8 (top curve) every 0.02. Dotted line represents the limit condition $n_{V} / N=T_{S} / T_{E}$. Inset: Time $\tau$ as a function of $\alpha$.

dependence of $\tau$ on the coupling between the excitable element and the ectopic focus, we have calculated the relative error for several values of parameter $\alpha$. Some of the results are presented in Fig. 10. Here $T_{E}=11$ and $T_{S}$ is changing over the adequate range for each case. We observe that, as $\alpha$ increases, the intersection of its corresponding curve with the limit curve $T_{S} / T_{E}$ occurs for smaller period ratio, which indicates a higher value of time $\tau$. The inset of Fig. 10 shows the obtained values of $\tau$ as a function of $\alpha$.

In summary, we have described a new scenario of competition between nonlinear oscillators that consists in their exported interaction through a common excitable element. Complex rhythms arise from such a coupling featuring some resemblances with the disordered records associated to some particular classes of cardiac arrhythmias. Specific statistical measures have been evaluated to discern both the role of the intrinsic firing periods of the competing oscillators as well as some intrinsic randomness in the system. The advantage with respect to cycle-map-based models [4] is that our concept of exported competition can be applied to any dynamical model exhibiting oscillatory and excitable behavior implementing competing units. A principal limitation is that we do not take into account the spatially extended structure, for example, of the cardiac tissue. Moreover, in this later context, a more realistic cardiac electrophysiological model would be necessary to reproduce clinical data, but our approach provides a very promising new perspective to analyze pure parasystole arhythmias.

This work was supported by the Ministerio de Ciencia y Tecnologia (Spain) under Project Nos. BFM2003-07850 and BQU2003-05042-C02-01. We also acknowledge financial support from the Comissionat per a Universitats i Recerca (Catalonia) under Project Nos. 2001SGR00045, 2001SGR00433, and 2001SGR00221. 
[1] A. Pikovsky, M. Rosenblum, and J. Kurths, Synchronization. A Universal Concept in Nonlinear Sciences (Cambridge University Press, Cambridge, 2003).

[2] P. Balenzuela and J. Garcia-Ojalvo, Chaos 15, 023903 (2005).

[3] Y. Soen, N. Cohen, D. Lipson, and E. Braun, Phys. Rev. Lett. 82, 3556 (1999).

[4] L. Glass, A. Goldberger, and J. Belair, Am. J. Physiol. 251, H841 (1986); M. Courtemanche, L. Glass, M. D. Rosengarten, and A. L. Goldberger, Am. J. Physiol. 257, H693 (1989).

[5] H.-X. Wang, R. de Paola, and W. I. Norwood, Phys. Rev. Lett. 70, 3671 (1993).

[6] V. Schulte-Frohlinde, Y. Ashkenazy, P. C. Ivanov, L. Glass, A.
L. Goldberger, and H. E. Stanley, Phys. Rev. Lett. 87, 068104 (2001).

[7] V. Schulte-Frohlinde, Y. Ashkenazy, A. L. Goldberger, P. C. Ivanov, M. Costa, A. Morley-Davies, H. E. Stanley, and L. Glass, Phys. Rev. E 66, 031901 (2002).

[8] C. Morris and H. Lecar, Biophys. J. 35, 193 (1981).

[9] B. S. Gutkin and G. B. Ermentrout, Neural Comput. 10, 1047 (1998); E. M. Izhikevich, Int. J. Bifurcation Chaos Appl. Sci. Eng. 10, 1171 (2000).

[10] V. Shusterman, B. Aysin, G. B. Ermentrout, B. London, and D. Schwartzman, J. Electrocardiol. 36, 219 (2003). 